\title{
Low-energy electron scattering by pyrazine
}

\author{
Carl Winstead and Vincent McKoy \\ A. A. Noyes Laboratory of Chemical Physics, California Institute of Technology, Pasadena, California 91125, USA
}

(Received 4 June 2007; published 18 July 2007)

\begin{abstract}
We report cross sections for low-energy elastic electron collisions with the diazabenzene molecule pyrazine, obtained from first-principles calculations. The integral elastic cross section exhibits three sharp peaks that are nominally shape resonances associated with trapping in the vacant $\pi^{*}$ molecular orbitals. Although the two lowest-energy resonances do in fact prove to be nearly pure single-channel shape resonances, the third contains a considerable admixture of core-excited character, and accounting for this channel coupling effect is essential to obtaining an accurate resonance energy. Such resonant channel coupling has implications for electron interactions with the DNA bases, especially the pyrimidine bases for which pyrazine is a close analog. In the absence of data on pyrazine itself, we compare our elastic differential cross section to measurements on benzene and find close agreement.
\end{abstract}

DOI: 10.1103/PhysRevA.76.012712

PACS number(s): 34.80.Bm, 34.80.Gs

\section{INTRODUCTION}

One of the most fundamental questions arising in lowenergy electron-molecule collisions is the nature of the scattering resonances that occur. Classifying resonances as elastic shape resonances, core-excited shape resonances, or Feshbach resonances is not merely a matter of systematizing them within an established nomenclature but of understanding their significance, since the available decay channels depend on the type of resonance involved [1]. On the other hand, any classification built on an independent-particle picture is necessarily inexact and may at times break down. Just as some bound electronic states of a many-electron system are poorly approximated by a single configuration of orbitals and require instead a multiconfigurational or "configuration interaction" (CI) description, so certain resonances may contain admixtures of different electronic configurations. Such a continuum $\mathrm{CI}$ is especially interesting in that its consequences will be directly observable in the scattering cross sections when the compound-state configurations that are involved correlate asymptotically to different states of the target molecule. In particular, mixing between an elasticchannel shape resonance and a core-excited shape resonance may enhance cross sections for electron-impact excitation.

In their pioneering studies of benzene and the azabenzenes, Nenner and Schulz [2] observed three (in the case of benzene itself, two) low-energy resonances in the elastic cross section, which they naturally interpreted as shape resonances arising from temporary trapping of the projectile electron in one of the three empty $\pi^{*}$ orbitals (the two lowest of which are degenerate in benzene). However, they also noted that the third resonance, which falls in the 4 to $5 \mathrm{eV}$ range, is above the threshold of the lowest excited states and so could mix with core-excited resonances built by coupling an excited state with a $\pi^{*}$ orbital of appropriate symmetry. In subsequent detailed investigations [3,4], Allan observed the decay of the "third" benzene resonance at $4.9 \mathrm{eV}$ into the first $\left({ }^{3} B_{1 u}\right)$ and second $\left({ }^{3} E_{1 u}\right)$ states (see also earlier work by Knoop [5]). These observations on benzene suggest that, as Nenner and Schulz posited, the third $\pi^{*}$ resonance of pyrazine, which lies at $4.1 \mathrm{eV}$ [2], is also likely to be of mixed elastic shape and core-excited character.
As well as influencing their decay channels, the character of the low-lying resonances influences (or should) their computational description. Calculations of low-energy electron collisions with polyatomics necessarily involve approximations, and in many-electron treatments of scattering, those approximations include the selection of a configuration space that provides a good description of the electron-molecule collision system but is of manageable size. In the case of a single-particle shape resonance, one can expect to account for the important correlation and polarization effects solely by providing for the response of the target charge density to the presence of the projectile electron. At long range, this is an electrostatic interaction that is largely captured in the dipole polarizability. At short range, and especially when a resonance extends the lifetime of the collision complex, target relaxation is more complicated, but to first order it is a matter of "reoptimizing" the occupied orbitals of the target in the presence of an extra electron, assuming a Hartree-Fock description of the target has been used. These considerations have inspired techniques for selecting configurations to represent polarization and correlation and for optimizing the virtual orbitals to accelerate the convergence of the configuration expansion [6-11], and those techniques have proven effective in various applications. It is noteworthy, however, that such polarization treatments only call for spinpreserving virtual excitations of the target molecule and do not emphasize excitations to physical states. Thus, if actual channel mixing with low-lying triplet states is occurring in pyrazine, conventional techniques for incorporating polarization effects into many-electron calculations are apt to overlook it. The situation appears even more problematic for oneelectron methods that treat polarization as a local attractive potential. In a one-electron picture, the $\pi^{*}$ orbitals occupy similar spatial regions and will presumably experience similar polarization shifts; however, coupling to core-excited resonances should affect different resonances to different degrees, depending on their proximity in energy to core-excited states of appropriate symmetry.

Our recent experience with elastic calculations on the purine and pyrimidine nucleobases of DNA and RNA [12-14] led us to reexamine our treatment of polarization effects. Consistently, we obtained fairly good energies for the lowest 


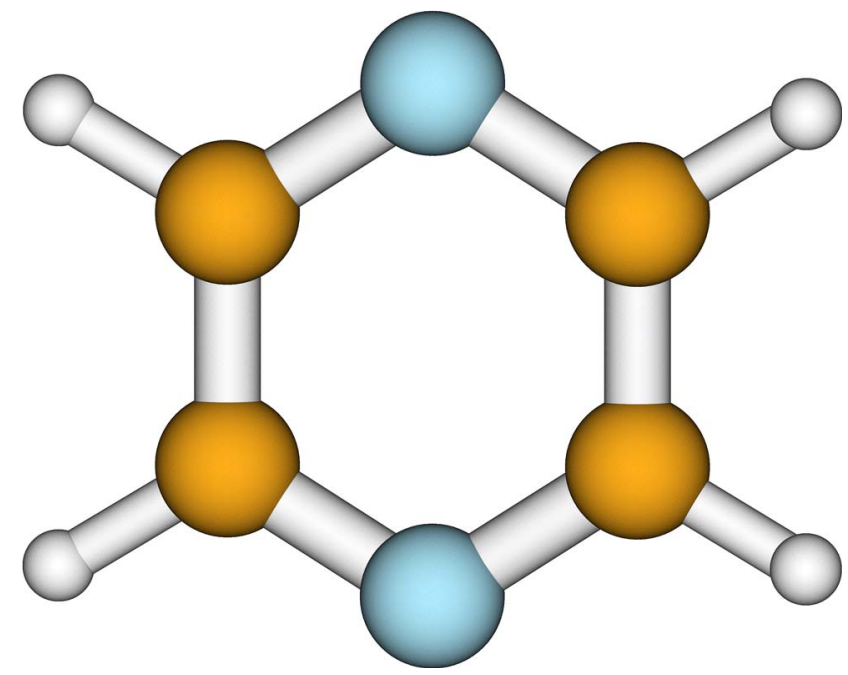

FIG. 1. (Color online) Structure of the pyrazine molecule. Nitrogen is blue (light), carbon brown (dark), and hydrogen white.

two resonances of the nucleobases in comparison to experiment $[15,16]$, but our results for the third resonance were always too high. Because the first two resonances involve slower projectiles and have longer lifetimes than the third, we would have expected just the opposite-i.e., that shortcomings in our description of polarization would be less important for the third resonance than for the first two. We chose pyrazine, a close analog of the pyrimidine bases, as a test case for further exploration because of its high symmetry and the availability of experimental data on the resonance positions. In this paper, we report differential and integral elastic cross sections for pyrazine obtained using an extended treatment of polarization that accounts for mixing of the third $\pi^{*}$ shape resonance with core-excited terms built on the low-lying triplet states, and we show that such mixing is indeed crucial to obtaining a good energy for that resonance. A preliminary account of some of this work was published previously [17].

The next section gives computational details. Results are presented and discussed in Sec. III.

\section{COMPUTATIONAL DETAILS}

The Schwinger multichannel (SMC) method $[18,19]$ and its implementation for parallel computers [20,21] have been described elsewhere, so we give here only details of the present calculations. Pyrazine (Fig. 1) is a diazabenzene molecule possessing $D_{2 h}$ point-group symmetry. We optimized the geometry at the level of second-order Möller-Plesset perturbation theory within the 6-31G(d) basis set as contained in the electronic structure program GAMESS [22], obtaining $r(\mathrm{CC})=1.3958 \AA, \quad r(\mathrm{CN})=1.3446 \AA, \quad r(\mathrm{CH})=1.0879 \AA$, $\angle(\mathrm{HCN})=116.637^{\circ}$, and $\angle(\mathrm{CCN})=122.358^{\circ}$. The ${ }^{1} A_{g}$ ground-state wave function was computed at the HartreeFock level in the $6-311++G(d, p)$ basis set, that is, the 6-311 $G$ basis set together with a $1 s 1 p$ diffuse supplement on $\mathrm{C}$ and $\mathrm{N}$, one diffuse $s$ Gaussian on $\mathrm{H}$, one $d$ function on $\mathrm{C}$ and $\mathrm{N}$, and one $p$ function on H. GAMESS default values were
TABLE I. Energies and symmetry labels for $\pi$ orbitals and lowlying excited states of pyrazine.

\begin{tabular}{|c|c|c|c|}
\hline \multicolumn{2}{|c|}{$\pi$ Orbitals } & \multicolumn{2}{|c|}{ Excited States } \\
\hline Label & Energy $(\mathrm{eV})$ & Label & Energy $(\mathrm{eV})$ \\
\hline$b_{3 g}\left(\pi_{1}\right)$ & -15.0 & $1^{3} B_{1 u}$ & 3.41 \\
\hline$b_{2 g}\left(\pi_{2}\right)$ & -11.9 & $1^{3} B_{3 u}$ & 4.06 \\
\hline$b_{1 g}\left(\pi_{3}\right)$ & -9.9 & $1{ }^{3} B_{2 u}$ & 4.08 \\
\hline$b_{3 u}\left(\pi_{1}^{*}\right)$ & 1.8 & $1{ }^{1} B_{3 u}$ & 5.11 \\
\hline$a_{u}\left(\pi_{2}^{*}\right)$ & 2.7 & $2{ }^{3} B_{1 u}$ & 5.13 \\
\hline$b_{2 g}\left(\pi_{3}^{*}\right)$ & 8.7 & $1{ }^{1} B_{2 u}$ & 5.90 \\
\hline
\end{tabular}

used for all exponents, and the $x^{2}+y^{2}+z^{2}$ combination of Cartesian $d$ Gaussians was excluded. The virtual orbitals from the Hartree-Fock calculation were transformed into modified virtual orbitals (MVOs) [23] via a +6 cationic Fock operator for subsequent use in the scattering calculations. For diagnostic purposes, we also carried out single-excitation CI calculations to determine energies of the low-lying singlet and triplet excited states, again in the $6-311++G(d, p)$ basis set. The excited-state energies so obtained are shown in Table I, which also shows Hartree-Fock energies for the occupied $\pi$ orbitals and the empty $\pi^{*}$ orbitals, the latter obtained from static-exchange calculations (neglecting polarization) in the $6-311+G(d, p)$ basis. The relevant orbitals are depicted in Fig. 2; the totally symmetric $\pi_{1}$ orbital is not shown because it is only very slightly involved in forming the low-lying excited states. Figures 1 and 2 were generated with MOLDEN [24].

For the scattering calculations incorporating polarization, we continued to employ the $6-311+G(d, p)$ basis set. In the five symmetries lacking $\pi^{*}$ resonances-that is, ${ }^{2} A_{g},{ }^{2} B_{1 g}$, ${ }^{2} B_{1 u},{ }^{2} B_{2 u}$, and ${ }^{2} B_{3 g}$-we treated polarization by allowing singlet-coupled single excitations from the 15 noncore occupied orbitals of the ground state into the 30 lowest-energy MVOs and coupling each such excitation with every additional MVO that produced the desired overall symmetry. We will refer to this 15-hole, 30-particle singlet-coupled excitation set as $15 h 30 p$ henceforward. As discussed in Sec. I, such a set of virtually-excited configurations is designed to allow the target molecule's charge density to relax in the presence of the projectile electron.

For the $\pi^{*}$-resonant symmetries ${ }^{2} B_{3 u},{ }^{2} A_{u}$, and ${ }^{2} B_{2 g}$, we experimented with different treatments of polarization. The

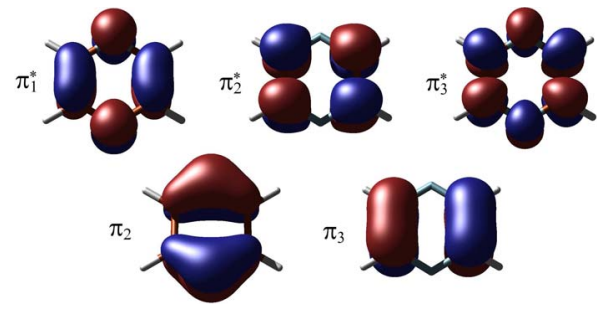

FIG. 2. (Color online) Occupied and vacant $\pi$ molecular orbitals of pyrazine involved in the formation of low-energy electronscattering resonances. 


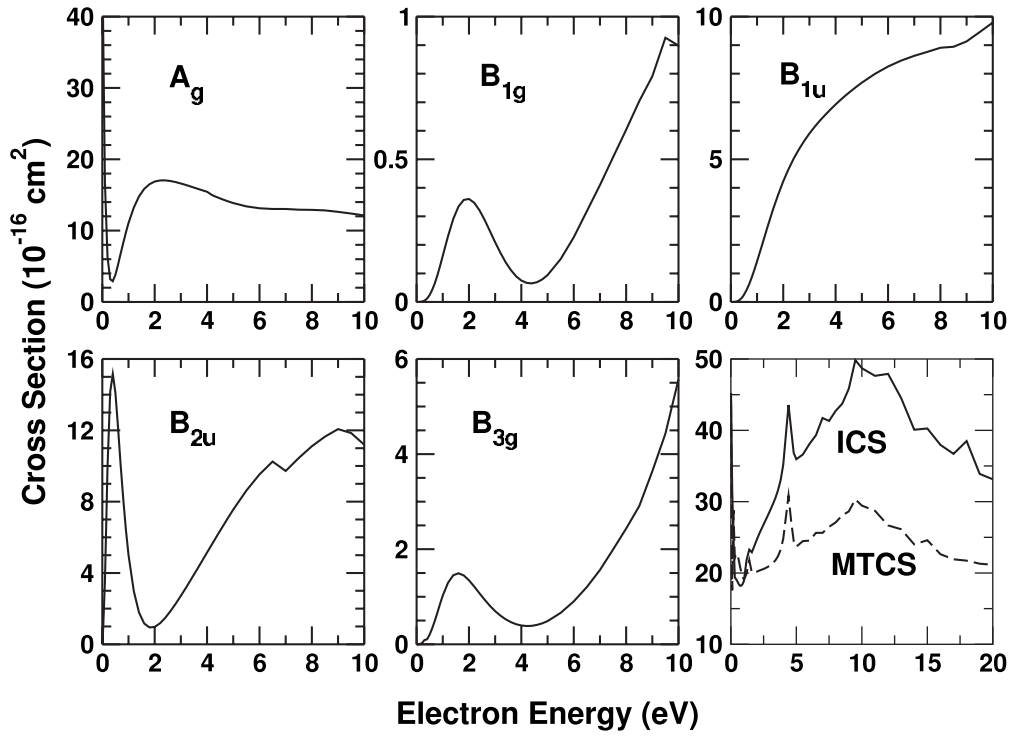

FIG. 3. Integral cross sections for scattering of electrons by pyrazine. The first five panels show the contributions to the integral elastic cross section from the non- $\pi^{*}$-resonant symmetries; the lower right panel shows the summed integral elastic cross section (ICS) and the momentum-transfer cross section (MTCS).
$15 h 30 p$ configuration set produced satisfactory results for ${ }^{2} B_{3 u}\left(\pi_{1}^{*}\right)$ and ${ }^{2} A_{u}\left(\pi_{2}^{*}\right)$ but not for ${ }^{2} B_{2 g}\left(\pi_{3}^{*}\right)$. Accordingly, we tried a larger set of closed-channel terms, with 15 "hole" and 43 "particle" orbitals used in singlet excitations together with triplet excitations from the three outermost occupied orbitals $\left(\pi_{2}\right.$, a $\sigma$ orbital, and $\left.\pi_{3}\right)$ into the 43 lowest MVOs $(15 h 43 p / 3 h 43 p)$. By deleting the triplet-coupled excitations from this calculation, we were also able to obtain $15 h 43 p$ results with only singlet-coupled target excitations. Finally, we increased the number of triplet-coupled excitations to include the six outermost valence orbitals while scaling back the size of the particle space to produce a $15 h 30 p / 6 h 30 p$ calculation. The number of doublet configuration state functions ranged from about 6000 for the $15 h 30 p{ }^{2} B_{1 g}$ calculation to about 10000 for each of the $15 h 43 p / 3 h 43 p$ calculations.

As will be discussed in the following section, including triplet-coupled excitations over-correlates the ${ }^{2} B_{3 u}$ and ${ }^{2} A_{u}$ resonances relative to the (Hartree-Fock) neutral ground state. Accordingly, our final differential and integral elastic cross sections were obtained by combining the $15 h 30 p$ scattering amplitudes for the non- $\pi^{*}$ symmetries with the $15 h 43 p$ amplitudes for ${ }^{2} B_{3 u}$ and ${ }^{2} A_{u}$ and the $15 h 30 p / 6 h 30 p$ amplitudes for ${ }^{2} B_{2 g}$.

\section{RESULTS AND DISCUSSION}

Integral elastic cross sections for the non- $\pi^{*}$-resonant symmetries are shown in Fig. 3. One interesting feature to note is the Ramsauer-Townsend minimum in ${ }^{2} A_{g}$ symmetry, confirmed not only by the minimum in the ${ }^{2} A_{g}$ component of the cross section but also by a sign change in the ${ }^{2} A_{g}$ eigenphase sum. However, the strong low-energy peak in ${ }^{2} B_{2 u}$ symmetry, which the eigenphase sum indicates to be nonresonant, is likely to obscure the Ramsauer-Townsend minimum in any measured cross section, as will the ${ }^{2} B_{3 u}\left(\pi_{1}^{*}\right)$ shape resonance described below. Low-energy maxima are also seen in ${ }^{2} B_{1 g}$ and ${ }^{2} B_{3 g}$ symmetries, though they are much weaker than that in ${ }^{2} B_{2 u}$.
Integral cross sections for the symmetries displaying lowenergy shape resonances are shown in Figs. 4 and 5. In these figures, we compare our resonance energies to the experimental resonance positions of Nenner and Schulz [2], which were obtained from derivative electron transmission measurements and are shown as horizontal bars in the figures, the energy width of each bar extending over the approximate width of the corresponding resonance feature (including vibrational structure) in the derivative spectrum. In Fig. 4, we see that the lowest-energy resonance, in ${ }^{2} B_{3 u}$ symmetry, occurs at about $0.15 \mathrm{eV}$ in the $15 h 43 p$ calculation, comfortably within the rather broad energy band determined by the vibrational progression in the experimental resonance. In the $15 h 30 p / 6 h 30 p$ calculation, which includes triplet-coupled excitations, the ${ }^{2} B_{3 u}$ state drops below $0 \mathrm{eV}$-that is, it becomes a bound anionic state, and the resonance peak disap-

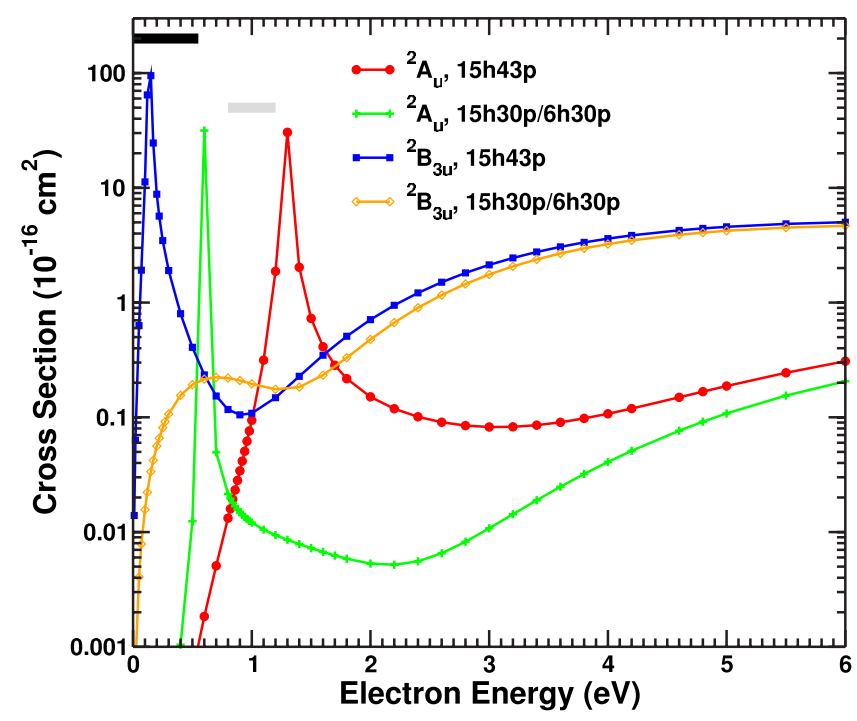

FIG. 4. (Color online) Partial cross sections for electronpyrazine scattering in the ${ }^{2} B_{3 u}$ and ${ }^{2} A_{u}$ symmetries. The horizontal bars (gray for ${ }^{2} A_{u}$ and black for ${ }^{2} B_{3 u}$ ) show resonance positions measured by Nenner and Schulz [2]. 


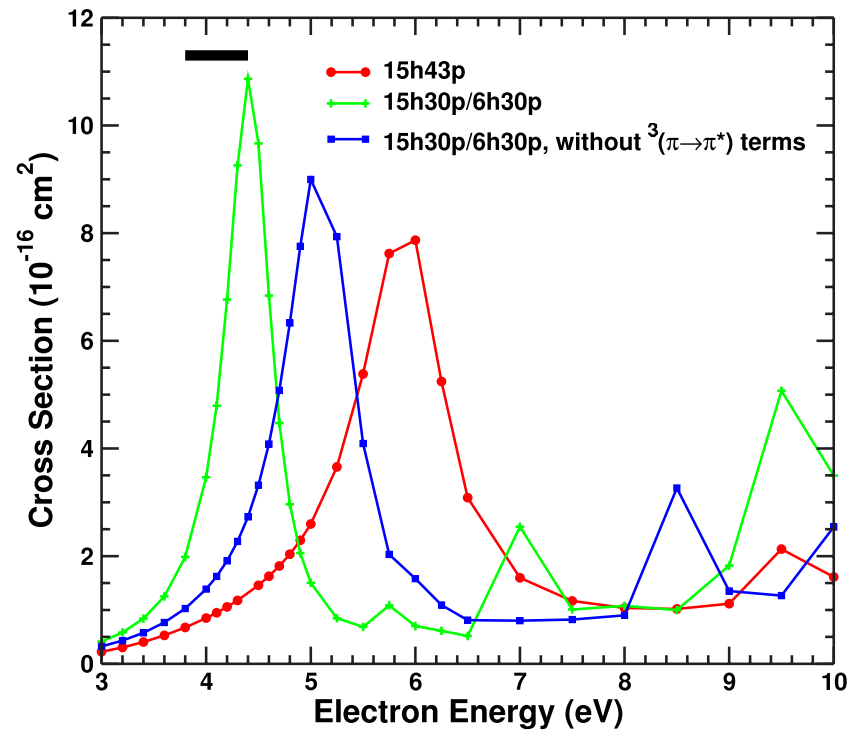

FIG. 5. (Color online) Partial cross sections for electronpyrazine scattering in ${ }^{2} B_{2 g}$ symmetry, obtained with different treatments of polarization (see text for discussion). The horizontal bar shows the resonance position measured by Nenner and Schulz [2].

pears from our fixed-nuclei cross section. This result is not necessarily inconsistent with experiment, since the lowestenergy features observed in the transmission spectrum may not correspond to the vibrational ground state of the anion. However, we can also see in the same figure that the second, ${ }^{2} A_{u}$ resonance is placed somewhat too low in the $15 h 30 p / 6 h 30 p$ calculation, indicating that we have overcorrelated it, and probably the ${ }^{2} B_{3 u}$ state as well, relative to the neutral ground state. Such overcorrelation can occur if the description of the temporary anion state is of better quality than the (Hartree-Fock) description of the neutral; in the present case, it probably reflects the inclusion of configurations such as $\left(\pi_{3} \rightarrow \pi_{2}^{*}\right) \otimes \pi_{3}^{*}$, which has $B_{3 u}$ symmetry and is doubly excited relative to the $\left(\pi_{3}\right)^{2}\left(\pi_{1}^{*}\right)^{1}$ configuration of the ${ }^{2} B_{3 u}$ resonance. We thus prefer the $15 h 43 p$ results for the ${ }^{2} B_{3 u}$ and ${ }^{2} A_{u}$ symmetries, even though the $15 h 43 p$ calculation places the ${ }^{2} A_{u}$ resonance a little too high in energy. On the whole, though, both the $15 h 43 p$ and the $15 h 30 p / 6 h 30 p$ calculations appear to describe these two resonances well, since errors on the order of a few tenths of an $\mathrm{eV}$ are fully to be expected at this level of approximation.

The situation is completely different for the ${ }^{2} B_{2 g}$ resonance, Fig. 5. In this case, the $15 h 43 p$ calculation, despite its comprehensive treatment of target relaxation through singletcoupled virtual excitations, yields a resonance energy nearly $2 \mathrm{eV}$ too high. As the $15 h 30 p / 6 h 30 p$ results show, nearly all of this error can be accounted for by including tripletcoupled excitations from the outer-valence orbitals. Moreover, as the third curve in Fig. 5 indicates, selectively deleting only the doublet configuration state functions built on the ${ }^{3}\left(\pi_{2} \rightarrow \pi_{1}^{*}\right),{ }^{3}\left(\pi_{3} \rightarrow \pi_{1}^{*}\right)$, and ${ }^{3}\left(\pi_{3} \rightarrow \pi_{2}^{*}\right)$ excitations that are responsible for the lowest-energy $\pi \rightarrow \pi^{*}$ triplet excited states of pyrazine shifts the resonance upward and away from the experimental position by nearly $1 \mathrm{eV}$, even though we are only removing 28 functions from the 9037-function
$15 h 30 p / 6 h 30 p{ }^{2} B_{2 g}$ variational space. Further experimentation (not shown in the figure) reveals that deleting the single configuration ${ }^{3}\left(\pi_{3} \rightarrow \pi_{1}^{*}\right) \otimes \pi_{2}^{*}$ raises the resonance energy by $0.3 \mathrm{eV}$. These last results demonstrate that it is not merely triplet-coupled excitations in general that are important to the description of this resonance, but specifically core-excited resonance terms built on the low-lying triplet states.

We can rationalize the difference in character between the two lowest-energy resonances and the third resonance on the basis that there are no core-excited configurations of appropriate symmetry nearby in energy with which the lowest resonances can mix, and they thus retain nearly pure singlechannel character. The third resonance, meanwhile, lies high enough in energy to interact with configurations such as ${ }^{3}\left(\pi_{3} \rightarrow \pi_{1}^{*}\right) \otimes \pi_{2}^{*}$ that arise from the low-lying triplet states and also belong to ${ }^{2} B_{2 g}$ symmetry. To provide semiquantitative support for this picture, we carried out small CI calculations on the anion states. Although lacking proper scattering boundary conditions, CI calculations in a compact basis set can give useful information about the electronic structure of "nearly bound," resonant states. Accordingly, we performed three diagnostic CI calculations on the doublet pyrazine $+e^{-}$system in the $6-311++G(d, p)$ basis set, with the neutral ground state times, respectively, the $\pi_{1}^{*}, \pi_{2}^{*}$, and $\pi_{3}^{*}$ MVOs as the reference configuration and including single through quadruple excitations within an active space comprising the three outermost valence occupied orbitals and the five lowest MVOs. The CI space thus included (but was not limited to) the $\pi_{2,3} \rightarrow \pi_{1-3}^{*}$ excitations. The resulting ${ }^{2} B_{3 u}$ and ${ }^{2} A_{u}$ wave functions were nearly pure singleconfiguration states, each with CI coefficient 0.97 for the reference configuration and each with an occupation number of 0.99 for the appropriate $\left(b_{3 u}\right.$ or $\left.a_{u}\right)$ natural orbital. In contrast, the ${ }^{2} B_{2 g}$ state had a CI coefficient of only 0.79 for the neutral ground state times $\pi_{3}^{*}$, and the occupation number of the $b_{2 g}$ natural orbital was only 0.66 , while natural orbitals having the symmetries of $\pi_{1}^{*}$ and $\pi_{2}^{*}$ also showed significant occupation: 0.44 for $b_{3 u}$ and 0.30 for $a_{u}$. Thus the ${ }^{2} B_{2 g}$ state, though still identifiable as a $\pi_{3}^{*}$ shape resonance, does in fact contain a large admixture of other configurations.

The integral elastic and momentum-transfer cross sections obtained by summing up our amplitudes for each irreducible representation of $D_{2 h}$ are shown in the bottom right panel of Fig. 3. The two lowest $\pi^{*}$ resonances, ${ }^{2} B_{3 u}$ and ${ }^{2} A_{u}$, are not fully resolved on the grid of energies used to make this plot, but the ${ }^{2} B_{2 g}$ resonance is prominent. The jagged structure at energies above the ${ }^{2} B_{2 g}$ resonance is typical of calculations of this kind, in which all channels except the elastic are treated as closed, giving rise to pseudoresonances above the excitation thresholds.

Differential cross sections (DCSs) for elastic electron scattering by pyrazine are shown at selected energies in Figs. 6 and 7. To our knowledge, the pyrazine DCS has not been measured, so we compare our results to DCS measurements by Cho and co-workers [25] for benzene. Overall, the agreement is quite satisfactory, especially considering that the DCS changes rapidly with energy and that we are comparing results for similar, but not identical, molecules. Indeed, our pyrazine DCSs are, at most energies, closer to the measured 


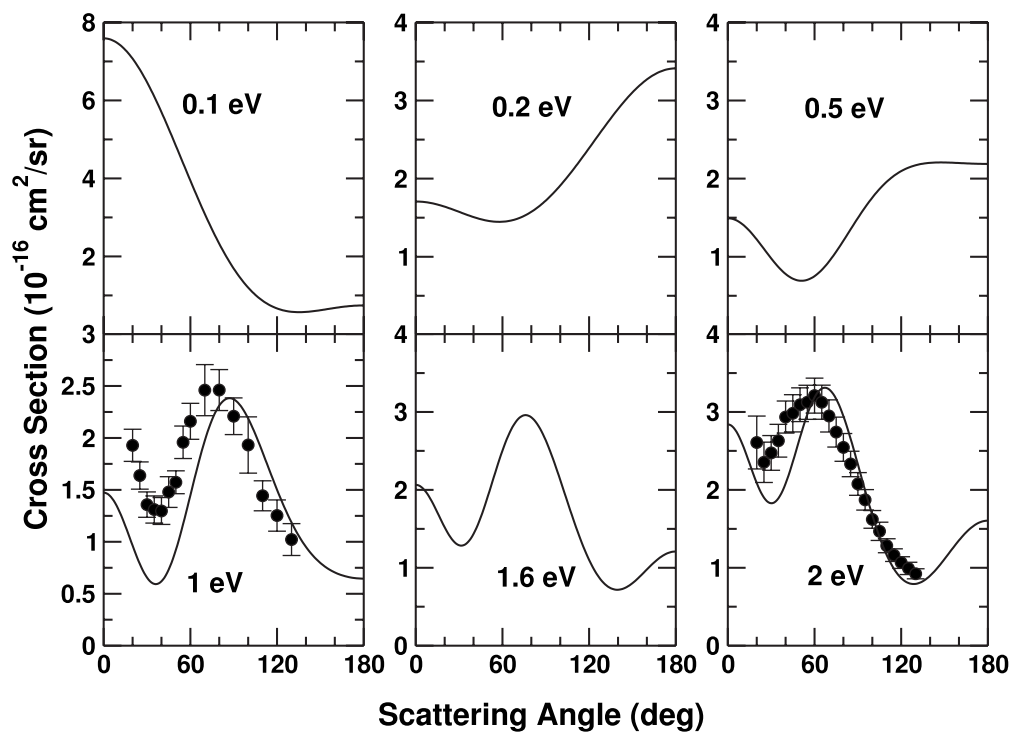

FIG. 6. Differential cross sections for elastic electron scattering by pyrazine at selected energies from the present calculation (solid curves) compared to measured cross sections for the related molecule benzene (circles with error bars). The benzene measurements are from Ref. [25].

benzene DCS than are either of two recent, high-level calculations on benzene itself [26,27], suggesting that the treatment of polarization effects was a limitation in those calculations. In particular, it is unlikely, given the prominence of the ${ }^{2} B_{2 g}$ resonance in the integral cross section, that we could have obtained reasonably accurate DCSs in the $4-6 \mathrm{eV}$ range without a treatment of polarization that allowed for coupling to core-excited triplet terms. The rapid variation in the shape of the DCS as a function of energy in the $0-5 \mathrm{eV}$ energy range appears to reflect the influence of the three low-energy resonances. The complete reversal from strong forward peaking at $0.1 \mathrm{eV}$ to backward peaking at $0.2 \mathrm{eV}$ is particularly striking. However, the slowness of the projectile and the narrowness of the ${ }^{2} B_{3 u}$ and ${ }^{2} A_{u}$ resonances make neglect of nuclear motion in our calculations a more serious limitation in this energy range than it is at $\sim 1 \mathrm{eV}$ and above; indeed, the electron-transmission measurements of Nenner and Schulz [2] show that the ${ }^{2} B_{3 u}$ resonance appears as multiple vibrational peaks between 0 and $0.5 \mathrm{eV}$. We nonetheless expect some qualitative, though likely not quantitative, resemblance may exist between our fixed-nuclei DCS and the vibrationally elastic DCS even below $1 \mathrm{eV}$.
The type of resonant channel mixing we have observed in pyrazine is likely to be a common phenomenon and to occur, in particular, not only in benzene and other azabenzenes, as suggested already by Nenner and Schulz [2], but also the nucleobases of RNA and DNA. Failure to provide for the possibility of such mixing is probably the main reason for large errors in the location of the "third" resonance of benzene in earlier calculations [26,27] and for similarly large errors in our results for the third resonance of the pyrimidine $[12,14]$ and purine $[13]$ nucleobases. As we have shown here for pyrazine, such errors can largely be eliminated, in allelectron calculations, by incorporating appropriate tripletcoupled virtual excitations into the variational basis set. In single-particle models that treat polarization as a local potential, it is less clear how to proceed, since the $\pi^{*}$ orbitals occupy similar spatial regions but undergo differential energy shifts due to their different degrees of mixing with coreexcited configurations.

Because mixed resonances decay not only into the electronically elastic channel but also into triplet states, their existence in the nucleobases suggests the possibility that, at energies above $4 \mathrm{eV}$, they may play a role in promoting the

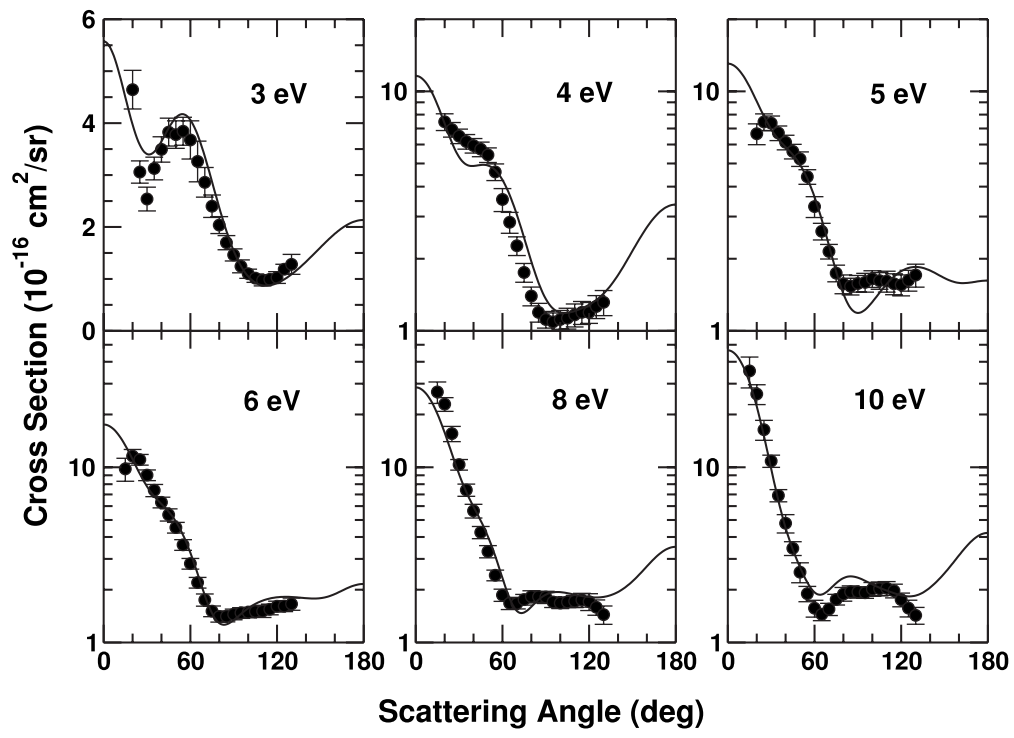

FIG. 7. As in Fig. 6, at higher energies. 
observed ability of slow electrons to damage DNA [28-31]. It is believed that the nucleobases were favored by natural selection in part because their ultraviolet photolysis is largely suppressed by conical intersections that rapidly return singlet excited states to high vibrational levels of the electronic ground state [32,33], whose excess energy can then be dissipated thermally before bond rupture occurs. Selective pressure to provide protection against electron-induced damage would likely have been weaker than that against photoninduced damage. Indeed, the first triplet state of thymine, in particular, is thought to play a role in the formation of cyclobutyl pyrimidine dimer lesions in DNA $[34,35]$ (although such lesions are also formed by photoexcitation of singlet states, e.g., Ref. [36]), and it is possible to speculate that triplet states might also play a role in strand breaking. Investigations of the triplet potential surfaces and of the electronimpact excitation cross sections would be useful.

\section{SUMMARY}

We have shown that inclusion of resonant channel coupling between the elastic channel and low-lying triplet states is essential to obtaining a good description of the ${ }^{2} B_{2 g}$ resonance in pyrazine, whereas the two lower-energy resonances are, to a good approximation, single-particle $\pi^{*}$ shape resonances. These results provide computational support for a hypothesis already proposed long ago by Nenner and Schulz [2] and account for discrepancies with experiment observed in prior calculations on related molecules that did not take account of such channel coupling [12-14,26,27]. Decay of mixed-character resonances in the nucleobases into triplet excited states may constitute a pathway for slow-electron damage to DNA. Differential cross sections obtained from the present work are in good agreement with measurements for the related molecule benzene.

\section{ACKNOWLEDGMENTS}

This work was supported by the Department of Energy, Office of Basic Energy Sciences. We gratefully acknowledge use of the computational resources of the Jet Propulsion Laboratory's Supercomputing and Visualization Facility.
[1] G. J. Schulz, Rev. Mod. Phys. 45, 423 (1973).

[2] I. Nenner and G. J. Schulz, J. Chem. Phys. 62, 1747 (1975).

[3] M. Allan, Helv. Chim. Acta 65, 2008 (1982).

[4] M. Allan, J. Electron Spectrosc. Relat. Phenom. 48, 219 (1989).

[5] F. W. E. Knoop, Ph.D. thesis, University of Leiden, 1972, as quoted in R. Azria and G. J. Schulz, J. Chem. Phys. 62, 573 (1975).

[6] B. I. Schneider, T. N. Rescigno, B. H. Lengsfield III, and C. W. McCurdy, Phys. Rev. Lett. 66, 2728 (1991).

[7] T. N. Rescigno, C. W. McCurdy, and B. I. Schneider, Phys. Rev. Lett. 63, 248 (1989).

[8] B. H. Lengsfield, III, T. N. Rescigno, and C. W. McCurdy, Phys. Rev. A 44, 4296 (1991).

[9] T. N. Rescigno, D. A. Byrum, W. A. Isaacs, and C. W. McCurdy, Phys. Rev. A 60, 2186 (1999).

[10] C. Winstead and V. McKoy, Phys. Rev. A 57, 3589 (1998).

[11] C. Winstead, V. McKoy, and M. H. F. Bettega, Phys. Rev. A 72, 042721 (2005).

[12] C. Winstead and V. McKoy, J. Chem. Phys. 125, 174304 (2006).

[13] C. Winstead and V. McKoy, J. Chem. Phys. 125, 244302 (2006).

[14] C. Winstead, V. McKoy, and S. d'A. Sanchez, J. Chem. Phys. (to be published).

[15] K. Aflatooni, G. A. Gallup, and P. D. Burrow, J. Phys. Chem. A 102, 6205 (1998).

[16] A. M. Scheer, K. Aflatooni, G. A. Gallup, and P. D. Burrow, Phys. Rev. Lett. 92, 068102 (2004).

[17] C. Winstead and V. McKoy, Phys. Rev. Lett. 98, 113201 (2007).

[18] K. Takatsuka and V. McKoy, Phys. Rev. A 24, 2473 (1981).

[19] K. Takatsuka and V. McKoy, Phys. Rev. A 30, 1734 (1984).

[20] C. Winstead and V. McKoy, Adv. At., Mol., Opt. Phys. 36, 183 (1996).
[21] C. Winstead and V. McKoy, Comput. Phys. Commun. 128, 386 (2000).

[22] M. W. Schmidt, K. K. Baldridge, J. A. Boatz, S. T. Elbert, M. S. Gordon, J. H. Jensen, S. Koseki, N. Matsunaga, K. A. Nguyen, S. J. Su, T. L. Windus, M. Dupuis, and J. A. Montgomery, J. Comput. Chem. 14, 1347 (1993).

[23] C. W. Bauschlicher, J. Chem. Phys. 72, 880 (1980).

[24] G. Schaftenaar and J. H. Noordik, J. Comput.-Aided Mol. Des. 14, 123 (2000).

[25] H. Cho, R. J. Gulley, K. Sunohara, M. Kitajima, L. J. Uhlmann, H. Tanaka, and S. J. Buckman, J. Phys. B 34, 1019 (2001).

[26] F. A. Gianturco and R. R. Lucchese, J. Chem. Phys. 108, 6144 (1998).

[27] M. H. F. Bettega, C. Winstead, and V. McKoy, J. Chem. Phys. 112, 8806 (2000).

[28] B. Boudaïffa, P. Cloutier, D. Hunting, M. A. Huels, and L. Sanche, Science 287, 1658 (2000).

[29] M. A. Huels, B. Boudaïffa, P. Cloutier, D. Hunting, and L. Sanche, J. Am. Chem. Soc. 125, 4467 (2003).

[30] F. Martin, P. D. Burrow, Z. Cai, P. Cloutier, D. Hunting, and L. Sanche, Phys. Rev. Lett. 93, 068101 (2004).

[31] R. Panajotovic, F. Martin, P. Cloutier, and L. Sanche, Radiat. Res. 165, 452 (2006).

[32] A. Broo, J. Phys. Chem. A 102, 526 (1998).

[33] A. L. Sobolewski and W. Domcke, Phys. Chem. Chem. Phys. 6, 2763 (2004), and references therein.

[34] I. G. Gut, P. D. Wood, and R. W. Redmond, J. Am. Chem. Soc. 118, 2366 (1996).

[35] F. Bosca, V. Lhiaubet-Vallet, M. C. Cuquerella, J. V. Castell, and M. A. Miranda, J. Am. Chem. Soc. 128, 6318 (2006).

[36] W. J. Schreier, T. E. Schrader, F. O. Koller, P. Gilch, C. E. Crespo-Hernández, V. N. Swaminathan, T. Carell, W. Zinth, and B. Kohler, Science 315, 625 (2007). 